
\title{
25 Research Square \\ Genetic Alterations of Esophageal Squamous Cell Carcinoma in Korean Patients
}

\section{Sunggyun Park}

Keimyung University School of Medicine

\section{Dongju Won}

Yonsei University College of Medicine

\section{Dae Joon Kim}

Yonsei University College of Medicine

\section{Seong Yong Park ( $\nabla$ sYPARKCS@yuhs.ac)}

Yonsei University College of Medicine

\section{Seung-Tae Lee}

Yonsei University College of Medicine

\section{Research Article}

Keywords: squamous cell carcinoma, esophageal cancer, whole exome sequencing, mutation signature

Posted Date: November 10th, 2021

DOI: https://doi.org/10.21203/rs.3.rs-1014087/v1

License: (c) (1) This work is licensed under a Creative Commons Attribution 4.0 International License. Read Full License 


\section{Abstract}

Background: Esophageal squamous cell carcinoma (ESCC) is one of the deadliest cancers in Korea, although its incidence is lower in Korea than in China and Japan. There are no data on genetic alterations associated with ESCC in Korea. Our study is the first report of the mutational landscape of ESCC in a Korean cohort.

Methods: We performed whole exome sequencing analysis of 43 ESCC tumor samples and germline DNA from normal esophageal mucosa or whole blood. Using electronic medical records, the patients' clinical characteristics were reviewed carefully.

Results: We found a mutually exclusive mutation pattern in NFE2L2-ZNF750 gene pairs. Non-silent variants of $K M T 2 D(M L L 2)$ were associated with a poor prognosis. An analysis to identify potentially druggable genes revealed NOTCH1 as a potential therapeutic target. We observed a high frequency of C:G $\rightarrow \mathrm{T}$ :A transitions regardless of smoking history. In our cohort, deconstruction of the mutation signature revealed enrichment of COSMIC single base substitution (SBS) 13, SBS 39, SBS 2, SBS 40, and SBS 1.

Conclusion: We did not find a Korean-specific signature of genetic alterations, but our data suggest that $K M T 2 D$ is a molecular prognostic marker. Further studies need to examine the role of KMT2D in ESCC prognosis due to our small sample size.

\section{Introduction}

Worldwide, esophageal cancer causes 544,076 deaths annually and is the sixth leading cause of cancer death. ${ }^{1}$ East Asia has the highest regional incidence rates for both men and women, in part because of the large burden in China. In 2017, there were 232,255 new cases of cancer in Korea, of which 2483 (1.1\% of all cancers) were esophageal cancer. ${ }^{2}$ The crude incidence rate of esophageal cancer was 4.8 cases per 100,000 population in 2017 . The age-standardized incidence rate decreased from 4.0 per 100,000 population in 1999 to 2.6 in 2017 , with annual percent changes of $-2.8 \%$ in men and $-1.3 \%$ in women. ${ }^{2}$ The incidence of esophageal cancer in Korea is relatively low compared with that in Japan and China, but the reasons for this are not clear. ${ }^{3}$ In $2013,90.2 \%$ of esophageal carcinoma cases was squamous cell carcinoma (ESCC) histologically, followed by adenocarcinoma (3\%). ${ }^{4}$ The proportions of localized and regional cancer tended to increase compared with that of distant cancer, and the 5-year relative survival rate of esophageal cancer improved from $14.0 \%$ in $1993-1995$ to $38.0 \%$ in $2013-2017 .{ }^{2}$ Nevertheless, the overall 5-year survival rate of patients with advanced cancer is less than $15 \%$, and novel therapeutic targets are necessary for better treatment strategies for ESCC. ${ }^{5}$

Several studies have examined the genetic alterations associated with ESCC in Chinese, Japanese, and Indian populations. ${ }^{5-10}$ For instance, a study of ESCC in a Chinese ESCC cohort of 139 reported known mutated genes, such as TP53, PIK3CA, and NOTCH1, and previously uncharacterized mutated genes including $F A T 1, F A T 2, Z N F 750$, and $K M T 2 D .^{7}$ Song et al. reported mutual exclusivity between mutations 
in NOTCH1 and PIK3CA, and that patients with NOTCH1 mutations had shorter survival times than patients without mutations. ${ }^{10}$ In a whole exome sequencing (WES) analysis of 144 Japanese patients with ESCC, Sawada et al. identified mutations in EP300 and TET2 that correlated with a shorter survival time $^{8}$ In a WES analysis of 28 Indians with ESCC, Kiran et al. reported higher frequencies of C:G $\rightarrow$ A:T transversion and mutation signature 4 compared to smokers and non-users of tobacco. ${ }^{5}$ However, the genomic alterations associated with ESCC in Korea are not well-characterized. In this study, we used WES to characterize the mutational landscape of ESCC in Korea.

\section{Results}

\section{Results of whole exome sequencing analysis}

Tumor and paired normal DNA from 43 ESCC patients was subjected to WES. The mean read depth of the target regions was $134.4 \subseteq$, and $99.0 \%$ of the target bases were covered by $>10$ independent reads after alignment. This identified 6,557 variants in 4,752 genes: 2,090 silent variants (synonymous and deep intronic variants) and 4,467 non-silent variants (Fig. 1). The non-silent variants include 3807 missense, 288 nonsense, 222 frameshift, 84 splicing, 51 in-frame, eight nonstop, and seven start codon variants. We found a median of 74 non-silent variants (range 18-307) per sample with a median tumor mutation burden of 2.47 (range $0.60-10.23$ ) non-silent variants/MB.

\section{Somatic mutation spectrum of esophageal squamous cell carcinoma}

Our ESCC cohort included the recurrently mutated genes TP53 (non-silent variants, $88 \%$ of samples), TTN (37\%), NFE2L2 (30\%), ZNF750 (23\%), NOTCH1 (21\%), FSIP2 (19\%), KMT2D (16\%), and LRP1B (16\%) (Fig.

1 , Supplementary Fig. S1). The top five affected oncogenic signaling pathways were TP53 (88\% of samples), NOTCH (58\%), RTK-RAS (53\%), Hippo (44\%), and NRF2 (37\%) (Supplementary Fig. S2). We identified NFE2L2 as a cancer driver gene with a single cluster, and all seven mutations were in that cluster based on the OncodriveCLUST algorithm run in maftools $(p<0.05) .{ }^{13}$ We performed Pfam annotation using maftools, and the top five mutated protein domains were COG5048, 7tm_1, FN3, Cadherin_repeat, and P53 (Supplementary Table S1). We observed a mutually exclusive mutation pattern in NFE2L2-ZNF750 gene pairs in ESCC (Fig. 2). In our cohort, NFE2L2 was mutated in $30 \%(13 / 43)$ of the samples and ZNF750 in 23\% (10/43) (Supplementary Fig. S1B, C).

\section{Clinical and prognostic relevance of each mutation in esophageal squamous cell carcinoma}

Table 1 summarizes the basic characteristics of the patients. The cohort contained 7 women and 35 men, with a median age of 70 (range 46-81) years. The median follow-up was 15.8 (range 2.3-31.4) months. Nodal metastasis was detected in $83.7 \%(7 / 43)$ of the patients and lympho-vascular invasion in $44.2 \%$ 
(19/43), confirmed pathologically. All patients were followed until either death or the last follow-up date. During the follow-up period, 7 (16.3\%) patients died and 13 (30.2\%) suffered recurrence (Table 1).

In the clinical enrichment analysis, there were no genes significantly associated with sex, a history of alcohol or tobacco consumption, the grade of tumor differentiation, the grade of tumor invasion, the presence of nodal metastasis, lymphovascular invasion, or perineural invasion.

In the overall survival analysis of the top 30 genes, non-silent variants of $K M T 2 D(M L L 2)$ were associated with a poor prognosis (hazard ratio $6.18, p<0.05$ ) (Fig. 3A). KMT2D was mutated in $16.3 \%(7 / 43)$ of the samples in our cohort (Supplementary Fig. S1D). The mortality rates in the mutated and non-mutated KMT2D groups were $42.9 \%$ and $11.1 \%$, respectively $(p=0.072)$. In the disease-free survival analysis of the top 30 genes, non-silent variants of $K M T 2 D(M L L 2)$ were associated with poor disease-free survival (hazard ratio 2.07) (Fig. 3B), but this was not significant $(p=0.191$ ).

In the disease-free survival analysis of the top 30 genes, non-silent variants of $\angle R P 1 B$ were associated with a greater likelihood of recurrence (hazard ratio 2.6), without statistical significance $(p=0.083)$ (Supplementary Fig. S3). The recurrence rates in the mutated and non-mutated $\angle R P 1 B$ groups were $71.4 \%$ and $22.2 \%$, respectively $(p<0.05)$. $\angle R P 1 B$ was mutated in $16.3 \%(7 / 43)$ of the samples in our cohort, and all non-silent variants of $\angle R P 1 B$ were missense variants (Supplementary Fig. S1E). 
Table 1

Basic characteristics of patients with esophageal squamous cell carcinoma (ESCC) enrolled in this cohort.

\begin{tabular}{|ll|}
\hline Characteristic & Our cohort $(\mathbf{n}=\mathbf{4 3})(\%)$ \\
\hline Sex & $7(16.3)$ \\
\hline Male & $36(83.7)$ \\
\hline Age (years) & \\
\hline Range & $46-81$ \\
\hline Median \pm standard deviation & $65.88 \pm 8.93$ \\
\hline Degree of differentiation & \\
\hline Well differentiated & $7(16.3)$ \\
\hline Moderately differentiated & $25(58.1)$ \\
\hline Poorly differentiated & $11(25.6)$ \\
\hline Depth of invasion & \\
\hline pT1b & $8(18.6)$ \\
\hline pT2 & $9(20.9)$ \\
\hline pT3 & $24(55.8)$ \\
\hline pT4b & $2(4.7)$ \\
\hline Nodal metastasis & \\
\hline Yes & $36(83.7)$ \\
\hline No & $7(16.3)$ \\
\hline Lymphovascular invasion & \\
\hline Yes & $19(44.2)$ \\
\hline No & $24(55.8)$ \\
\hline Death & \\
\hline Yes & \\
\hline No & \\
\hline Recurrence & $(30.2)$ \\
\hline
\end{tabular}




\begin{tabular}{|ll|}
\hline Characteristic & Our cohort $(\mathbf{n}=\mathbf{4 3})(\%)$ \\
\hline No & $30(69.8)$ \\
\hline Alcohol consumption & \\
\hline Current drinker & $13(30.2)$ \\
\hline Ex-drinker & $23(53.5)$ \\
\hline Non-drinker & $7(16.3)$ \\
\hline Smoking status & \\
\hline Current smoker & $13(30.2)$ \\
\hline Ex-smoker & $22(51.2)$ \\
\hline Non-smoker & $8(18.6)$ \\
\hline
\end{tabular}

\section{Identification of potentially druggable genes}

To identify potential druggable targets, we screened genes bearing non-silent variants detected in at least $20 \%$ of the samples against FDA-approved antineoplastic drugs in DGIdb. ${ }^{14}$ This revealed NOTCH1 as a potential therapeutic target. In our cohort, NOTCH1 was mutated in $21 \%(9 / 43)$ of the samples (Supplementary Fig. S1F).

\section{Mutation signatures associated with smoking history}

In this study, seven samples were from non-smokers. The median mutation burden was 2.47 and 2.43 non-silent variants/MB in smokers and non-smokers, respectively. We observed a high frequency of C:G $\rightarrow \mathrm{T}$ :A transitions regardless of smoking history. Further, deconstruction of the mutation signature of our cohort revealed the enrichment of COSMIC single base substitution (SBS) 13, SBS 39, SBS 2, SBS 40, and SBS 1 (Fig. 4). We did not observe distinct signatures associated with ESCC in patients with a history of smoking or alcohol consumption.

\section{Discussion}

This study investigated the mutational landscape of ESCC in a Korean cohort. We identified several known and novel variants in ESCC. The observed median tumor mutation burden of non-silent variants (2.47/MB) was comparable to the reported mutation load of $1.9-3 / \mathrm{MB} \cdot{ }^{5-9,15}$ We identified several frequently mutated genes, such

as TP53, TTN, NFE2L2, ZNF750, NOTCH1, FSIP2, and KMT2D, which have been reported in ESCC. Mutated genes commonly found in over $10 \%$ of the ESCC samples in previous reports and this study are 
described in Supplementary Table S2. Except for TTN, NFE2L2, and FSIP2, most of these genes had nonsense, frameshift, or splicing variants, suggesting their tumor suppressor roles (Fig. 1).

The NRF2 pathway consisting of NFE2L2, KEAP1, and CUL3 was genetically deregulated in $37.2 \%$ of our cohort (Supplementary Fig. S1). NFE2L2 encodes a transcription factor that induces the production of a cytoprotective enzyme in response to oxidative stress, whereas the KEAP1/CUL-dependent proteasomal mechanism degrades the NFE2L2 protein under non-stressed circumstances. ${ }^{16}$ All NFE2L2 variants in our cohort involved two hot spots within the DLG and ETGE motifs that bind to KEAP1, as observed in a Japanese cohort. ${ }^{8}$ These mutations are thought to contribute to tumor development by stabilizing the NFE2L2 protein. ${ }^{17}$ There were NFE2L2 mutations in $9.6-16.7 \%$ of the cases in previous reports. ${ }^{6,8,18}$ Recently, in a Chinese cohort of 508 patients, NFE2L2 mutations were reported to be significantly associated with a worse prognosis of ESCC. ${ }^{19}$ But there was no significant association between the prognosis of ESCC and NFE2L2 mutations based on our data.

$Z N F 750$, an epidermal differentiation regulator, is thought to be an ESCC tumor suppressor gene, ${ }^{7,8,15}$ as supported by our data, which showed that most ZNF750 variants were null variants (Fig. 1). ZNF750 is mutated in $3.9-16.7 \%$ of ESCC cases. ${ }^{6,18,20}$ In Japanese ESCC datasets, ZNF750 variants were positively associated with the APOBEC signature, ${ }^{8}$ but not in our study $(p=0.069)$.

We observed a mutually exclusive mutation pattern in NFE2L2-ZNF750 gene pairs in ESCC, although this mutual exclusivity has not been implicated in any type of cancer. ${ }^{21}$ Mutual exclusivity has been widely observed in cancer genomes and there are two major hypotheses associated with mutually exclusive mutated genes in cancers: the functional redundancy in downstream pathways and synthetic lethality hypotheses. ${ }^{22}$ Because these two genes do not share the same pathway, the mutually exclusive mutation in NFE2L2-ZNF750 gene pairs supports the synthetic lethality hypothesis.

$\mathrm{NOTCH} 1$ is frequently disrupted by loss-of-function mutations, implying that the loss of NOTCH pathway activity is critical for the growth of tumor cells with squamous differentiation characteristics. ${ }^{23}$ NOTCH1 is mutated in about $16 \%$ of cases. $6,8,18$ There are several reports that NOTCH1 is associated with a poor prognosis, and its mutations are mutually exclusive with PIK3CA mutations. ${ }^{5,10,24}$ However, there was no significant association between the prognosis of ESCC and NOTCH1 mutations based on our data. Nevertheless, the analysis to identify potentially druggable genes revealed $\mathrm{NOTCH} 1$ as a potential therapeutic target.

$K M T 2 D$ is tumor suppressor gene that encodes histone methyltransferase and promotes the transcriptional activation of target genes by modifying histone $\mathrm{H} 3$ lysine 4 trimethylation (H3K4me3) ${ }^{25}$ KMT2D is mutated in $11-19 \%$ of ESCC cases, ${ }^{6,8,18}$ and Kaplan-Meier survival analysis showed that patients with non-silent variants of $K M T 2 D$ had poor overall survival. Abulajiang et al. reported an association between $K M T 2 D$ expression and the prognosis of ESCC, with KMT2D overexpression predicting poor clinical outcomes and facilitating ESCC tumor progression. ${ }^{26}$ 
$\angle R P 1 B$ is putative tumor suppressor gene that encodes low-density lipoprotein receptor-related protein $1 \mathrm{~b}^{27}$. $\angle R P 1 B$ is frequently deleted in various tumors and was deleted in $20.8 \%$ of a Japanese cohort ${ }^{8}$ and mutated in $25 \%$ of an Indian cohort. ${ }^{5} A$ study of $\angle R P 1 B$ gene expression in ESCC showed that $42.9 \%$ of primary esophageal cancer cases have homozygous $\angle R P 1 B$ deletions, and $\angle R P 1 B \mathrm{mRNA}$ expression was frequently silenced in cell lines without homozygous deletions (37.8\%). ${ }^{28}$ Bisulfite-PCR analysis and sequencing showed that $\angle R P 1 B$-nonexpressing cells without homozygous deletions were highly methylated at a $\angle R P 1 B \mathrm{CpG}$ island. Recently, Brown et al. reported better immune checkpoint inhibitor treatment responses in a group with likely pathogenic/pathogenic variants in $\angle R P 1 B$ genes compared with a group carrying variant of unknown significance, indicating that mutations in the $L R P 1 B$ gene have significant implications for the prognosis and treatment of multiple cancer types. ${ }^{27}$ All $L R P 1 B$ variants in our study were missense variants. Further studies of their expression and methylation are needed to clarify the roles of these $\angle R P 1 B$ missense mutations.

Kiran et al. showed that tobacco chewers had a higher frequency of mutation signature 4 than did smokers and non-users of tobacco in an Indian ESCC cohort. ${ }^{5}$ There were no tobacco chewers in our study and no distinct signatures associated with ESCC in patients with a smoking history.

Our study is the first report of the mutational landscape of ESCC in a Korean cohort. It provides insight into molecular alterations in ESCC in Koreans and reveals potential candidates for therapeutic targeting. Our data suggest that $K M T 2 D$ is a molecular prognostic marker. Further studies need to examine the role of KMT2D in ESCC prognosis due to the small size of our cohort. Additionally, copy number and expression studies of ESCC in Koreans are needed, as none were conducted here.

\section{Conclusion}

ESCC is one of the deadliest cancers in Korea, although its incidence in Korea is lower than in China and Japan. There are no data on genomic alterations associated with ESCC in Korea. Our study is the first report of the mutational landscape of ESCC in a Korean cohort. We found a mutually exclusive mutation pattern in NFE2L2-ZNF750 gene pairs. Non-silent variants of KMT2D (MLL2) were associated with a poor prognosis. An analysis to identify potentially druggable genes revealed $\mathrm{NOTCH} 1$ as a potential therapeutic target. We observed a high frequency of $\mathrm{C}: \mathrm{G} \rightarrow \mathrm{T}$ :A transitions regardless of smoking history. Further, deconstruction of the mutation signature of our cohort revealed the enrichment of COSMIC SBS 13 , SBS 39, SBS 2, SBS 40, and SBS 1. In summary, this study characterized the genomic alterations of ESCC with highly mutational heterogeneity, which provides the understanding of the molecular pathophysiology of ESCC, and it can be useful for finding potential therapeutic target in the aspect of precision medicine.

\section{Materials And Methods}

\section{Patients and samples}


We performed WES analysis of 43 ESCC tumor samples and germline DNA from normal esophageal mucosal tissue or whole blood obtained during surgical resection. The patients' clinical characteristics were reviewed using electronic medical records (Table 1). The study protocol was approved by the Institutional Review Board of Yonsei University College of Medicine (approval no. IRB 4-2018-1210). All subjects gave informed consent when they were enrolled and all methods were performed in accordance with the relevant guidelines and regulations of Institutional Review Board of Yonsei University College of Medicine.

\section{Whole exome sequencing analysis}

A WES DNA library was constructed using an Agilent SureSelect V6-Post kit (Agilent Technologies, Santa Clara, CA) with genomic DNA extracted from fresh-frozen tissue using the Qiagen DNeasy Blood \& Tissue kit (QIAGEN, Hilden, Germany). Genomic DNA was sheared into 150-200-bp fragments using an ultrasonic sonicator. These sheared fragments were end-repaired, phosphorylated, adenylated at the $3^{\prime}$ ends, and ligated using paired-end adaptors. The hybridization reaction was done at $65^{\circ} \mathrm{C}$ for 16 hours using a DNA library with added biotinylated RNA library bait (Agilent Technologies). Cluster amplification was performed according to the manufacturer's protocol (Illumina, San Diego, CA). Paired-end sequencing was performed on an Illumina NovaSeq 6000 system (Illumina) by Macrogen Inc. (Seoul, South Korea).

Raw reads acquired in FASTQ format were trimmed using the Trimmomatic tool in paired-end mode to increase the mapping accuracy and specificity. ${ }^{11}$ These were mapped against the reference genome hg19 (GRCh37) using the Burrows-Wheeler aligner with default parameters. Binary alignment map (bam) files were further processed using GATK (Genome Analysis Toolkit, Broad Institute, Cambridge, MA), which included the removal of duplicates using MarkDuplicates in Picard, IndelRealigner, and BaseRecalibrator. High-confidence somatic single-nucleotide variants (SNVs) were filtered using HaplotypeCaller, Mutect2, VarScan, and Pindel and annotated using AnnoVar and VEP. The variant call format (vcf) files were converted into mutation annotation format (maf) files using vcf2maf tools [Cyriac Kandoth (2020); mskcc/vcf2maf: vcf2maf v1.6.19, doi:10.5281/zenodo.593251].

Oncoplot, lollipop plots of somatic SNVs, the identities of genes with mutually exclusive variants and cancer driver genes, and actionable therapeutic target and mutational signatures were generated using the $\mathrm{R}$ package maftools. ${ }^{12}$ To predict clinically actionable therapeutic targets, the Drug-Gene Interaction Database (DGIdb) was accessed, and the "Druggable Genome" category was used to identify potentially druggable targets in ESCC. ${ }^{5}$

\section{Statistical analysis}

The overall and disease-free survival distributions were described with Kaplan-Meier curves, and statistical significance was calculated using the log-rank test in STATA 16. Overall survival was evaluated from the time of diagnosis to death or the last follow-up. Censored cases were defined as patients who lost contact during follow-up and who were still alive at the end of the study. Disease-free survival was 
evaluated from the time of diagnosis to death, the recurrence of ESCC, or the last follow-up. Censored cases were defined as patients who were still alive and had no recurrent tumors at the end of the study.

Clinical enrichment analysis was conducted using the R package maftools, with which pairwise and group-wise Fisher's exact tests can be performed to find differentially enriched genes for clinical features such as sex and determine whether metastasis has occurred, the patient survives, or the cancer has recurred.

\section{Declarations}

\section{Data availability}

The data that support the findings of this study are available from the corresponding author upon reasonable request.

\section{Acknowledgments}

This research was supported by the Basic Science Research Program through the National Research Foundation of Korea (NRF) funded by the Ministry of Education (2019R111A1A01055513).

\section{Author contributions}

S.P analyzed, curated, interpreted the data, and wrote the paper. S.Y.P and S.T.L conceptualized this research, review, editing this paper, and supervised entire project. S.Y.P acquired the funding. All authors reviewed the results and approved the final version of the manuscript.

\section{Competing interests}

The authors declare no competing interests.

\section{References}

1. Sung, H. et al. Global Cancer Statistics 2020: GLOBOCAN estimates of incidence and mortality worldwide for 36 cancers in 185 countries. Ca Cancer J Clin 71, 209-249 (2021).

2. Hong, S. et al. Cancer statistics in Korea: Incidence, mortality, survival, and prevalence in 2017. Cancer Res Treat 52, 335-350 (2020).

3. Kim, D. J., Park, S. Y. \& Hong, M. H. Esophageal squamous cell carcinoma, diagnosis and treatment. 363-376 (2020) doi:10.1007/978-981-15-4190-2_19. 
4. Shin, A. et al. Trends in incidence and survival of esophageal cancer in Korea: Analysis of the Korea Central Cancer Registry Database. J Gastroen Hepato/ 33, 1961-1968 (2018).

5. Mangalaparthi, K. K. et al. Mutational landscape of esophageal squamous cell carcinoma in an Indian cohort. Frontiers Oncol 10, 1457 (2020).

6. Gao, Y.-B. et al. Genetic landscape of esophageal squamous cell carcinoma. Nat Genet 46, 1097$1102(2014)$.

7. Lin, D.-C. et al. Genomic and molecular characterization of esophageal squamous cell carcinoma. Nat Genet 46, 467-473 (2014).

8. Sawada, G. et al. Genomic landscape of esophageal squamous cell carcinoma in a Japanese population. Gastroenterology 150, 1171-1182 (2016).

9. Song, Y. et al. Identification of genomic alterations in oesophageal squamous cell cancer. Nature 509, 91-95 (2014).

10. Song, B. et al. Mutually exclusive mutations in NOTCH1 and PIK3CA associated with clinical prognosis and chemotherapy responses of esophageal squamous cell carcinoma in China. Oncotarget 7, 3599-3613 (2015).

11. Bolger, A. M., Lohse, M. \& Usadel, B. Trimmomatic: a flexible trimmer for Illumina sequence data. Bioinformatics 30, 2114-2120 (2014).

12. Mayakonda, A., Lin, D.-C., Assenov, Y., Plass, C. \& Koeffler, H. P. Maftools: efficient and comprehensive analysis of somatic variants in cancer. Genome Res 28, 1747-1756 (2018).

13. Tamborero, D., Gonzalez-Perez, A. \& Lopez-Bigas, N. OncodriveCLUST: exploiting the positional clustering of somatic mutations to identify cancer genes. Bioinformatics 29, 2238-2244 (2013).

14. Griffith, M. et al. DGIdb: mining the druggable genome. Nat Methods 10, 1209-1210 (2013).

15. Cheng, C. et al. Genomic analyses reveal FAM84B and the NOTCH pathway are associated with the progression of esophageal squamous cell carcinoma. GigaScience 5, 1 (2016).

16. Shibata, T. et al. Cancer related mutations in NRF2 impair its recognition by Keap1-Cul3 E3 ligase and promote malignancy. Proc National Acad Sci 105, 13568-13573 (2008).

17. Sporn, M. B. \& Liby, K. T. NRF2 and cancer: the good, the bad and the importance of context. Nat Rev Cancer 12, 564-571 (2012).

18. Du, P. et al. Comprehensive genomic analysis of oesophageal squamous cell carcinoma reveals clinical relevance. Sci Rep-uk 7, 15324 (2017).

19. Cui, Y. et al. Whole-genome sequencing of 508 patients identifies key molecular features associated with poor prognosis in esophageal squamous cell carcinoma. Cell Res 30, 902-913 (2020).

20. Zhang, L. et al. Genomic analyses reveal mutational signatures and frequently altered genes in esophageal squamous cell carcinoma. Am J Hum Genetics 96, 597-611 (2015).

21. Kandoth, C. et al. Mutational landscape and significance across 12 major cancer types. Nature 502, 333-339 (2013). 
22. Deng, Y. et al. Identifying mutual exclusivity across cancer genomes: computational approaches to discover genetic interaction and reveal tumor vulnerability. Brief Bioinform 20, 254-266 (2017).

23. Ntziachristos, P., Lim, J. S., Sage, J. \& Aifantis, I. From fly wings to targeted cancer therapies: A centennial for notch signaling. Cancer Cell 25, 318-334 (2014).

24. Ogawa, R. et al. NOTCH1 expression predicts patient prognosis in esophageal squamous cell cancer. Eur Surg Res 51, 101-107 (2014).

25. Kerimoglu, C. et al. Histone-methyltransferase MLL2 (KMT2B) is required for memory formation in mice. J Neurosci 33, 3452-3464 (2013).

26. Abudureheman, A. et al. High MLL2 expression predicts poor prognosis and promotes tumor progression by inducing EMT in esophageal squamous cell carcinoma. J Cancer Res Clin 144, 1025-1035 (2018).

27. Brown, L. C. et al. LRP1B mutations are associated with favorable outcomes to immune checkpoint inhibitors across multiple cancer types. J Immunother Cancer 9, e001792 (2021).

28. Sonoda, I. et al. Frequent silencing of Low Density Lipoprotein Receptor-Related Protein 1B (LRP1B) expression by genetic and epigenetic mechanisms in esophageal squamous cell carcinoma. Cancer Res 64, 3741-3747 (2004).

\section{Figures}

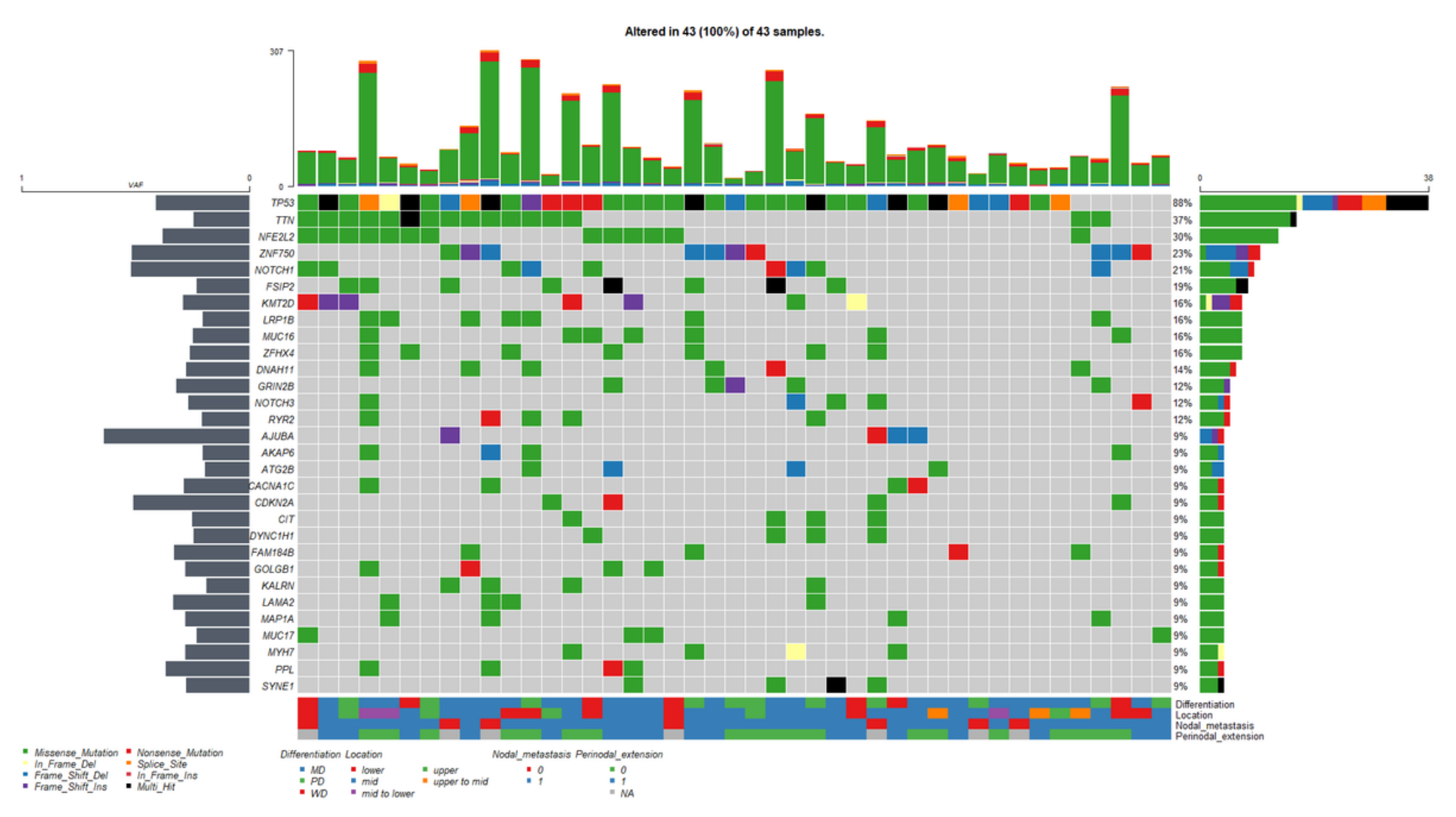


Figure 1

Oncoplots of the mutational landscape of esophageal squamous cell carcinoma (ESCC). The following characteristics of the top 30 genes are included: (top panel) the mutation load per MB, (middle panel) sample details including sample ID, tumor differentiation grade, tumor location, presence of nodal metastasis, and perinodal extension. Each column represents a sample and each row depicts a gene. Somatic mutations are colored according to the mutation type, and the right panel gives the percentages of samples harboring mutations.

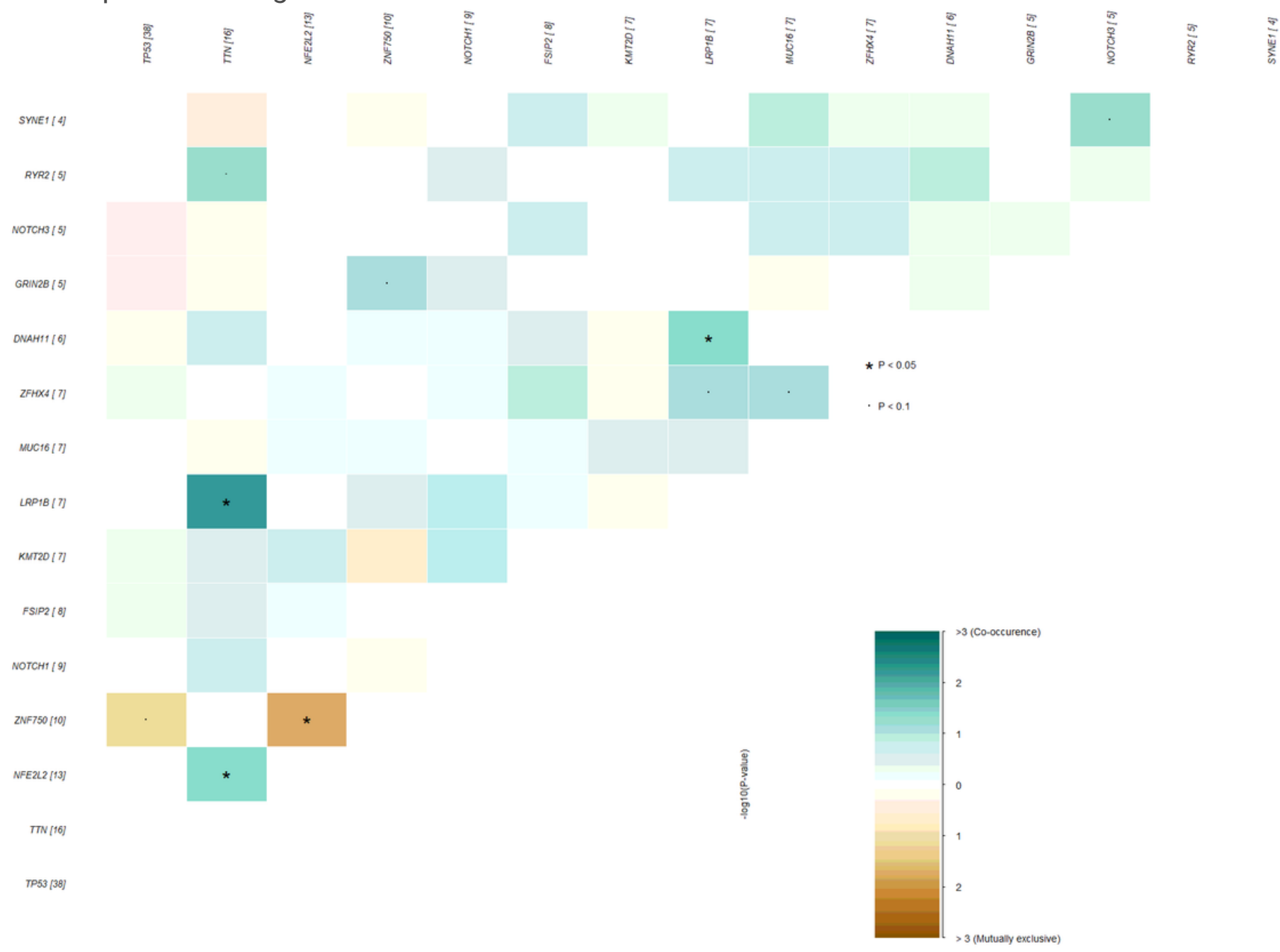

Figure 2

Somatic interactions of the top 15 genes in ESCC, using pair-wise Fisher's exact tests to detect significant gene pairs. 
A.

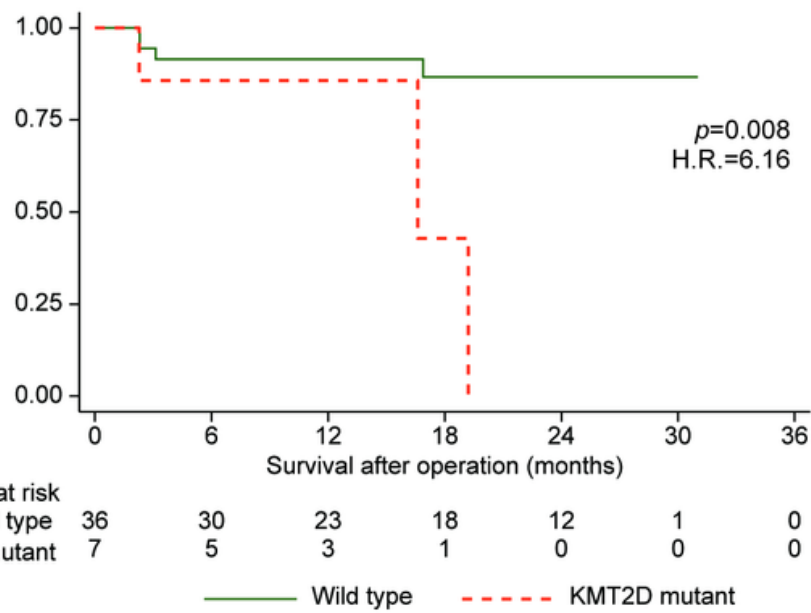

B.

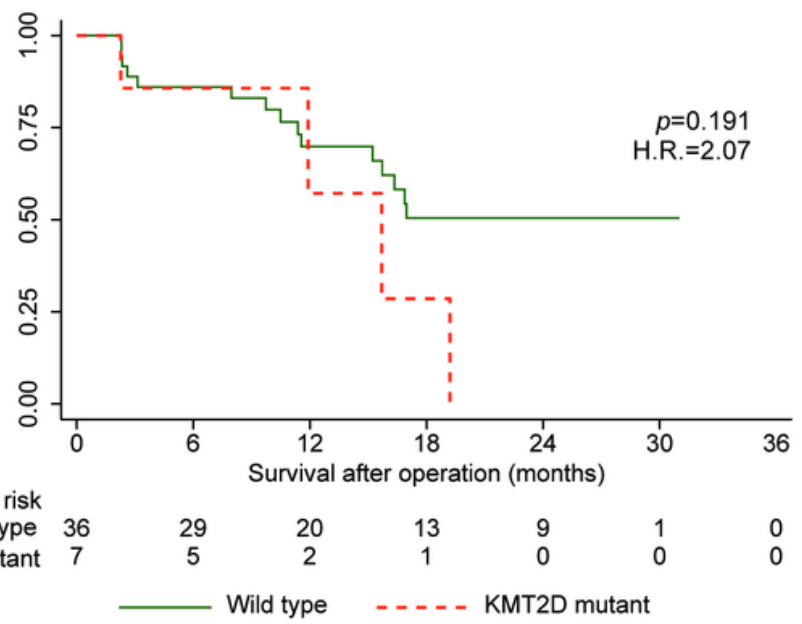

\section{Figure 3}

Overall survival (A) and recurrence (B) according to KMT2D mutation status. Kaplan-Meier survival curves of patients with KMT2D mutations or the wild type in our cohort. The p-values were computed using the log-rank test. 

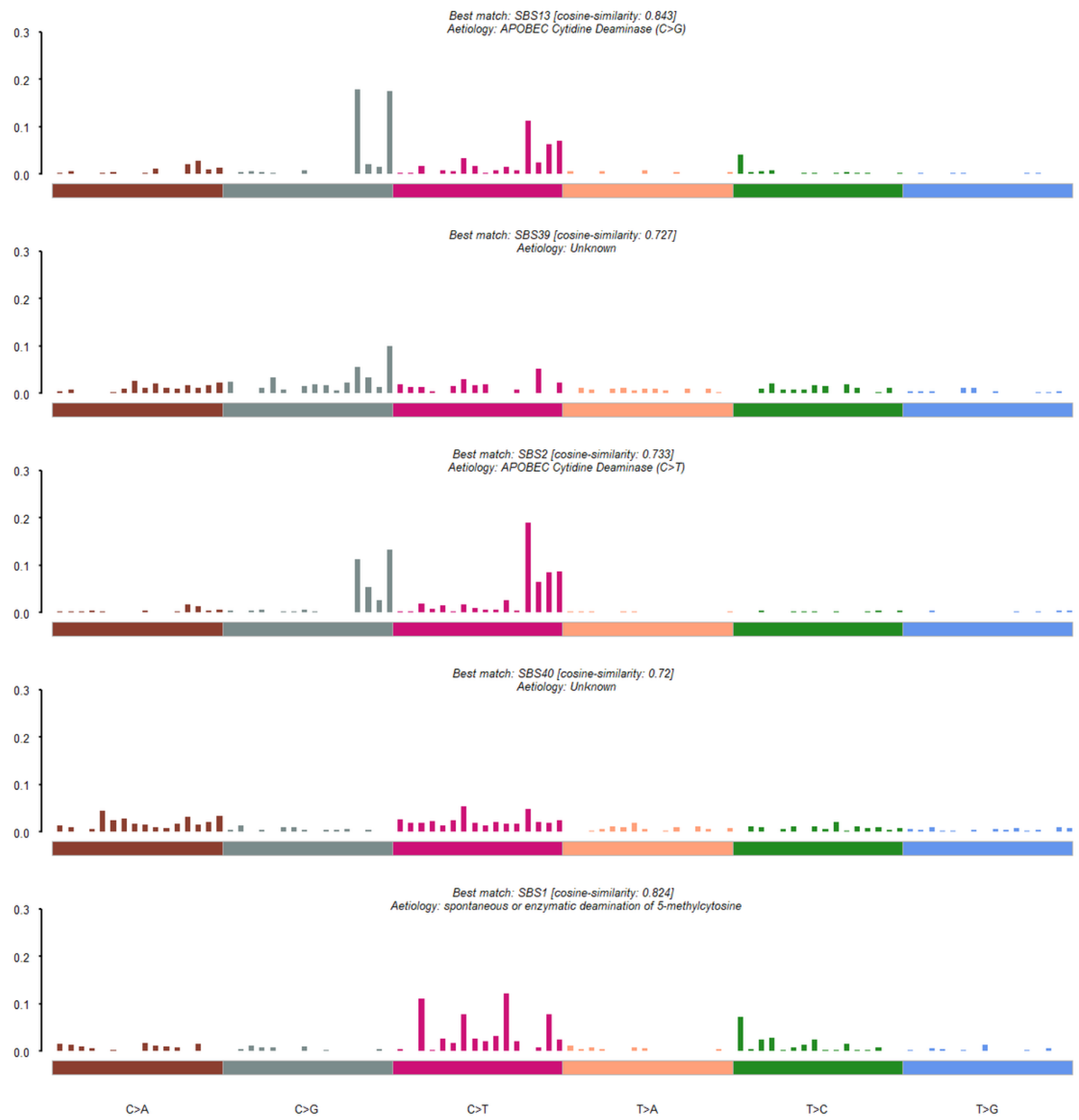

Figure 4

COSMIC single base substitution (SBS) signatures of our cohort.

\section{Supplementary Files}

This is a list of supplementary files associated with this preprint. Click to download. 
- Supplementalinfromation20211013.docx

Page 16/16 